\title{
Uso de Leersia hexandra (Poaceae) en la fitorremediación de suelos contaminados con petróleo fresco e intemperizado
}

\author{
Alfredo Arias-Trinidad ${ }^{1 *}$, María del Carmen Rivera-Cruz ${ }^{1}$, Antonio Roldán-Garrigós ${ }^{2}$, Lorenzo \\ Armando Aceves-Navarro ${ }^{1}$, Roberto Quintero-Lizaola ${ }^{3}$ \& Javier Hernández-Guzmán ${ }^{4}$ \\ 1. Colegio de Postgraduados, Campus Tabasco, Laboratorio de Microbiología, 86500 H. Cárdenas, Tabasco, México; \\ alfredo.arias8@yahoo.com.mx,mariari@colpos.mx, laceves@colpos.mx \\ 2. Consejo Superior de Investigaciones Científicas (CEBAS-CSIC), Campus Universitario de Espinardo, 30100 Murcia, \\ España; aroldan@cebas.csic.es \\ 3. Colegio de Postgraduados, Campus Montecillo, Montecillo, 56230 Texcoco, Estado de México, México; \\ roquintero@colpos.mx \\ 4. Universidad Juárez Autónoma de Tabasco, Ciencias Biológicas, 86150 Villahermosa, Tabasco, México; \\ jhernandez-guzman@hotmail.com
}

\author{
Recibido 02-II-2016. Corregido 21-VII-2016. Aceptado 18-VIII-2016.
}

\begin{abstract}
Use of Leersia hexandra (Poaceae) for soil phytoremediation in soils contaminated with fresh and weathered oil. The oil industry has generated chronic oil spills and their accumulation in wetlands of the state of Tabasco, in Southeastern Mexico. Waterlogging is a factor that limits the use of remediation technologies because of its high cost and low levels of oil degradation. However, Leersia hexandra is a grass that grows in these contaminated areas with weathered oil. The aim of the study was to evaluate the bacteria density, plant biomass production and phytoremediation of L. hexandra in contaminated soil. For this, two experiments in plastic tunnel were performed with fresh (E1) and weathered petroleum (E2) under waterlogging experimental conditions. The E1 was based on eight doses: 6000, 10 000, 30000, 60 000, 90000, 120000, 150000 and 180000 mg.kg-1 dry basis (d. b.) of total petroleum hydrocarbons fresh (TPH-F), and the E2, that evaluated five doses: $14173,28400,50598,75492$ and $112142 \mathrm{mg} . \mathrm{kg}^{-1} \mathrm{~d}$. b. of total petroleum hydrocarbons weathered (TPH-W); a control treatment with $2607 \mathrm{mg} \cdot \mathrm{kg}^{-1} \mathrm{~d}$. b. was used. Each experiment, with eight replicates per treatment, evaluated after three and six months: a) microbial density of total free-living nitrogen-fixing bacteria (NFB) of Azospirillum (AZP) and Azotobacter group (AZT), for viable count in serial plate; b) dry matter production (DMP), quantified gravimetrically as dry weight of L. hexandra; and c) the decontamination percentage of hydrocarbons (PDH) by Soxhlet extraction. In soil with TPH-F, the NFB, AZP y AZT populations were stimulated five times more than the control both at the three and six months; however, concentrations of 150000 and $180000 \mathrm{mg} \cdot \mathrm{kg}^{-1} \mathrm{~d}$. b. inhibited the bacterial density between 70 and $89 \%$. Likewise, in soil with TPH-W, the FNB, AZP and AZT inhibitions were $90 \%$, with the exception of the $14173 \mathrm{mg} \cdot \mathrm{kg}^{-1} \mathrm{~d}$. b. treatment, which stimulated the NFB and AZT in 2 and 0.10 times more than the control, respectively. The DMP was continued at the six months in the experiments, with values of 63 and $89 \mathrm{~g}$ in fresh and weathered petroleum, respectively; had no significant differences with the control $(\mathrm{p} \leq 0.05)$. The PDH reached values of 66 to $87 \%$ both TPH-F and TPH-W at six months, respectively. These results demonstrated the ability the L. hexandra rhizosphere to stimulate the high NFB density, vegetal biomass production and phytoremediation of contaminated soils (with fresh and weathered petroleum), in a tropical waterlogging environment. Rev. Biol. Trop. 65 (1): 21-30. Epub 2017 March 01.
\end{abstract}

Key words: Azotobacter, Azospirillum, tropical grass, weathered petroleum, phytoremediation.

En México, la región sur comprende los estados de Tabasco, Campeche, Chiapas, Yucatán y Quintana Roo, así como parte de Guerrero, Oaxaca y Veracruz, reconocidos productores de petróleo. En marzo 2016, la región tuvo una producción de $376 \mathrm{Mbd}$ (miles de barriles diarios de producción de hidrocarburos líquidos) y 1331 MMpcd (millones de pies cúbicos diarios) (PEMEX, 2016). Sin embargo, aunado a esta producción el detrimento ambiental 
es considerable, pues sólo del 2000 al 2014 se han generado 846 emergencias ambientales, de las cuales el $10.3 \%$ se registraron en el estado de Tabasco (PROFEPA, 2014). La mayoría de los sitios contaminados con petróleo y sus derivados, se encuentran en humedales o sitios anegados, que limitan el uso de especies fitorremediadoras bajo estas condiciones ambientales (García-López, Zavala-Cruz \& Palma-López, 2006; Hernández-Rivera et al., 2011). El petróleo según el tiempo que permanece en el ambiente puede ser fresco (PF) o intemperizado (PI), por lo que su efecto tóxico, cancerígeno, mutagénico y genotóxico puede variar (Caravaca \& Roldán 2003; Rainho, Corrêa, Mazzei, Aiub, \& Felzenszwalb, 2013). Los hidrocarburos del PF pueden volatilizarse, disolverse en la solución del suelo, ser absorbidos en la superficie del suelo o ser transformados en otros compuestos por diferentes procesos químicos y bioquímicos (Peña, Trasar, Gil, \& Leirós, 2007; Wang, Zhan, Zhou, \& Lin, 2010). Con la modificación de las propiedades químicas del suelo y los cambios en los ciclos bioquímicos, esto origina la mortalidad de la flora y fauna, (OMI, 2005; HernándezCastellanos et al., 2013). Por otro lado, el PI es aquel que permanece en la intemperie más de 10 años (Capó, 2007; Zavala-Cruz, TrujilloCapistrán, Ortiz-Ceballos, \& Ortiz, 2013), y tiene efectos tóxicos más graves que el $\mathrm{PF}$, por los hidrocarburos recalcitrantes, los cuales presentan mayor tiempo y grado de degradación, al estabilizarse en el medio ambiente (Fernández et al., 2006; Maletic, Dalmacija, Roncevic, Agbaba, \& Garcina, 2011).

La fitorremediación es una tecnología que utiliza plantas para degradar, extraer, acumular y estabilizar contaminantes orgánicos e inorgánicos del suelo (Lum, Ngwa, Chikoye, \& Suh, 2014). El sistema radical de las gramíneas puede alcanzar profundidades de hasta $2.7 \mathrm{~m}$, así como una alta tolerancia a condiciones climáticas extremas, altas concentraciones de salinidad o acidez, y presencia de metales pesados (Paz-Alberto \& Sigua, 2013; Mganga, 2014; Chávez-Rodríguez, 2015), lo que permite la estimulación y el establecimiento de poblaciones de microflora tanto en rizoplano como rizósfera, que contribuyen a la degradación de moléculas orgánicas (Uren, 2001; Bisht et al., 2015). L. hexandra Swartz (Oryzeae, Poaceae) es una especie endémica del estado de Tabasco, es una opción interesante para la evaluar su desempeño en la descontaminación de suelos, debido a su crecimiento en humedales, por su sistema de raíz rizomatoso y largos estolones fibrosos, que le permiten sobrevivir en periodos de sequía, lluvias y nortes (tormentas con fuertes vientos) (Aparicio et al., 2007; Liu, Duan, Zhang, Zhu, \& Xiaoyan, 2011; ShaoHong, Xue-Hong, Jie, Yi-Nian, \& Chen, 2013). El objetivo de este estudio, fue determinar la capacidad fitorremediadora de $L$. hexandra a partir de la densidad de bacterias fijadoras de nitrógeno de vida libre de la rizósfera, la producción de biomasa seca (MS), y la descontaminación de los hidrocarburos (DS) del suelo con petróleo fresco (PF) e intemperizado (PI) en condiciones de anegamiento.

\section{MATERIALES Y MÉTODOS}

Para el bioensayo, se recolectó (abril 2012, época de sequía) suelo de la zona, caracterizado como tipo Gleysol (GL) de dos sitios, a partir del horizonte superficial $(0-30 \mathrm{~cm})$. El primer sitio y primera muestra de suelo, no presentó contaminación antrópica derivada de la industria petrolera, y se localiza en el ejido Arroyo Hondo Santa Teresa, Cárdenas, Tabasco, México (17 $\left.59^{\prime} 53^{\prime \prime} \mathrm{N}-93^{\circ} 25^{\prime} 01^{\prime \prime} \mathrm{W}\right)$ (GL1). El segundo sitio, presentó un derrame crónico de petróleo que, a pesar de haber sido restaurado, han persistido concentraciones de hidrocarburos del petróleo. Sin embargo, por el tiempo de exposición a los factores ambientales, se consideró un suelo contaminado con altos contenidos de hidrocarburos de PI; este sitio se localiza en el ejido José Narciso Rovirosa, a dos kilómetros al suroeste del complejo procesador de gas La Venta, Huimanguillo, Tabasco (184'37' $\mathrm{N}-94^{\circ} 2$ '28” W). De este sitio se recolectaron cinco muestras de suelos (GL2, GL3, GL4, GL5 y GL6), a los que se le determinó y registró conforme NOM-021-RECNAT-2000 
CUADRO 1

Propiedades físicas y químicas de los suelos recolectados de los sitios de estudio

TABLE 1

Physical and chemical properties of soil collected from the study sites

\begin{tabular}{cccccc} 
Suelo & Textura & $\mathrm{pH}$ & Materia orgánica (\%) & Nitrógeno total $(\%)$ & Hidrocarburos totales del petróleo $\left(\mathrm{mg} . \mathrm{kg}^{-1} \mathrm{~b} . \mathrm{s}.\right)$ \\
GL1 $^{1}$ & Arcillosa & 6.9 & 10.18 & 0.4 & 2607 \\
GL2 $^{2}$ & Arcillosa & 6.09 & 21.93 & 0.6 & 14173 \\
GL3 & Arcillosa & 5.71 & 23.00 & 0.5 & 28400 \\
GL4 & Arcillosa & 5.36 & 16.39 & 0.6 & 50598 \\
GL5 & Arcillosa & 6.49 & 23.59 & 0.5 & 75492 \\
GL6 & Arcillosa & 6.14 & 16.39 & 0.5 & 112142 \\
\hline
\end{tabular}

1. Suelo testigo (hidrocarburos de origen biogénico) generado por quema de vegetación.

2. Suelos del sitio dos (GL2-GL6) presentan concentraciones de petróleo que superan los límites máximos permisibles estipulados en la NOM-138-SEMARNAT/SSA1-2008 (DOF, 2013).

(DOF, 2002) (Cuadro 1), norma mexicana que establece las especificaciones de fertilidad, salinidad y clasificación de suelos. Estudios, muestreo y análisis.

Los ejemplares de L. hexandra Swartz (Oryzeae, Poaceae) se recolectaron del sitio contaminado. Se preparó tres almácigos en contenedores de plástico con suelo GL1 y se colocó en un túnel de plástico (16 m largo, $6 \mathrm{~m}$ ancho y $2.5 \mathrm{~m}$ de alto) en las instalaciones del Colegio de Postgraduados, Campus-Tabasco. De los tallos maduros, se cortaron estolones de cinco centímetros de longitud con raíces, y se sembraron 60 días antes de establecer los experimentos, para el traslado a las unidades experimentales. La altura de las plántulas establecidas en el experimento fue en promedio de $12 \pm 3.7 \mathrm{~cm}$ de altura (30 días después de sembrarlos en los almácigos).

Diseños experimentales: El bioensayo constó de dos experimentos (E1 y E2) con arreglo bifactorial (dosis de HTP y tiempo de evaluación) y se estableció en agosto del 2012, en base a los requerimientos del Protocolo 208 de la Organización para la Cooperación y Desarrollo Económico (OECD, 2003) modificado (Rivera-Cruz \& Trujillo-Narcia, 2004), que permite identificar los síntomas de estrés que genera una sustancia química, en la emergencia y crecimiento de plántulas y plantas. Con ocho tratamientos en el E1 y cinco tratamientos en el E2, con ocho repeticiones por tratamiento, ambos completamente al azar. Para obtener concentraciones de 6000, 10000, 30000, $60000,90000,120000,150000$ y 180000 mg.kg-1 b. s. HTPF del E1. Los tratamientos del E2 con $14173,28400,50598,75492$ y 112142 mg.kg ${ }^{-1}$ b. s. HTPI correspondieron a los suelos GL2, GL3, GL4, GL5 y GL6, respectivamente. Para ambos experimentos, el suelo G1 (2607 mg. $\mathrm{kg}^{-1}$ b. s. HTP) se consideró como tratamiento testigo. Cada unidad experimental (U.E.) contenía $1.5 \mathrm{~kg}$ de suelo (Fig. 1).

En el E1, el suelo de cada unidad experimental del GL1 se molió y tamizó previo a ser mezclado con las cantidades $12,15,45,90,108$, 144, 210 y 252 g PF, y se homogenizó con una varilla de vidrio, la mezcla se colocó en contenedores de vidrio $(15 \times 14.5 \mathrm{~cm})$. En el caso de los suelos del E2 (GL2, GL3, GL4, GL5 y GL6) se molieron, tamizaron y homogenizaron antes de ser depositados en los contenedores, posteriormente las unidades experimentales se colocaron en el túnel de plástico de manera aleatoria (Fig. 1). En ambos experimentos, a cada unidad experimental se trasplantó una planta de $L$. hexandra y se aplicó riego con una probeta de plástico de $1000 \mathrm{~mL}$ cada 24 $\mathrm{h}$ para mantener condiciones de anegamiento del suelo (ocho centímetros arriba del suelo). A los tres y seis meses, aleatoriamente se destruyeron cuatro unidades experimentales por tratamiento de cada experimento, se recolectó 


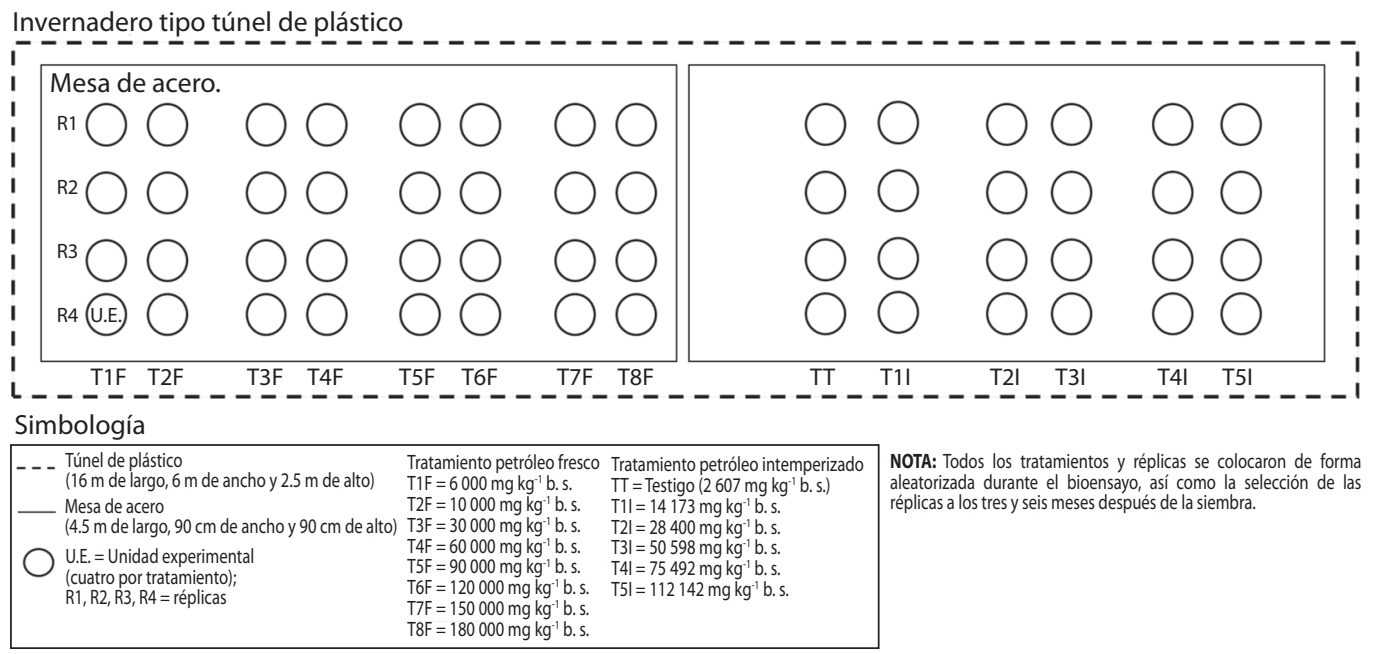

Fig. 1. Diseño experimental. / Fig. 1. Experimental design.

suelo con rizósfera para evaluar las poblaciones de bacterias fijadoras de nitrógeno de vida libre totales (BFN), la producción de MS y DSC del suelo de L. hexandra.

Densidad de bacterias fijadoras de nitrógeno: Se evaluó con el método de dilución seriada y determinación de cuenta viable en cajas de Petri (Madigan, Martinko, Dunlap \& Clark, 2009). Se utilizó los medios de cultivos: carbón combinado para las BFN (Rennie, 1981), rojo congo para el grupo de AZP (Reinhold et al., 1987), y el medio Ashby para el grupo AZT (Döbereiner, Marriel \& Nery, 1966).

Porcentaje de descontaminación: El porcentaje se determinó con la concentración de HTP, que se basó en la cuantificación por la NMX-AA-134-SCFI-2006 (DOF, 2006).

Producción de materia seca: Para determinar la producción de MS (biomasa seca foliar y radical), las plantas se secaron en horno a $70{ }^{\circ} \mathrm{C}$ durante $48 \mathrm{~h}$ y se pesaron en balanza analítica (Atrya, modelo TE4101).

Se utilizó el software SPSS para determinar el análisis de varianza y la prueba de medias entre los tratamientos (Tukey $\mathrm{p} \leq 0.05$ ).

\section{RESULTADOS}

Densidad de bacterias fijadoras de nitrógeno: A los tres y seis meses, las poblaciones de BFN presentaron una estimulación en la rizósfera de $L$. hexandra con diferencias significativas entre los tratamientos (Tukey $\mathrm{p} \leq 0.05$ ) (Cuadro 2). En el PF el tratamiento con 120000 mg. $\mathrm{kg}^{-1}$ b. s. HTPF presentó la mayor densidad de BFN, a los tres y seis meses con $90 \times 10^{2}$ y $26 \times 10^{3}$ UFC. $^{-1}$ rizósfera seca (r.s.), respectivamente. Sin embargo, las densidades de AZP y AZT entre tratamientos y tiempo de evaluación fueron irregulares. En PI las BFN y AZT alcanzaron la mayor densidad en suelo con $112142 \mathrm{mg} \cdot \mathrm{kg}^{-1}$ b. s. HTPI con valores de 41 × $10^{2}$ y 22 × $10^{2}$ UFC.g $^{-1}$ r.s., respectivamente. Por el contrario, a los seis meses disminuyó la densidad de BFN y AZT, al incrementar los contenidos de HTPI de los suelos, a excepción del suelo con $14173 \mathrm{mg} . \mathrm{kg}^{-1}$ b. s. HTPI $\left(22 \times 10^{2}\right.$ y $16 \times 10^{2}$ UFC.g $^{-1}$ r.s., respectivamente), las densidades de AZP fueron inhibidas en ambos tiempos con el PI.

Producción de la materia seca: $L$. hexandra presentó diferencias significativas tanto en los tipos de petróleo como en los tiempos de evaluación en relación al suelo testigo (Tukey, $\mathrm{p} \leq 0.05$ ). La mayor producción de MS 
CUADRO 2

Densidad de bacterias fijadoras de nitrógeno y grupos (Azospirillum y Azotobacter) de la rizósfera de Leersia hexandra de suelos contaminados con petróleo fresco e intemperizado, a los tres y seis meses

TABLE 2

Total nitrogen-fixing bacteria and groups (Azospirillum y Azotobacter) density in rhizosphere of Leersia hexandra in soil with fresh and weathered oil

\begin{tabular}{|c|c|c|c|c|c|c|}
\hline \multirow{2}{*}{$\begin{array}{l}\text { HTP (mg.kg }{ }^{-1} \text { b. s.) } \\
\text { Fresco }\end{array}$} & \multicolumn{3}{|c|}{ Tres meses $\left(10^{-1}\right.$ UFC.g $\mathrm{g}^{-1}$ b. s. $)$} & \multicolumn{3}{|c|}{ Seis meses $\left(10^{-1}\right.$ UFC.g $g^{-1}$ b. s. $)$} \\
\hline & BFN & AZP & AZT & $\mathrm{BFN}$ & AZP & AZT \\
\hline Testigo & $150 \mathrm{~b}$ & $21 b$ & $199 \mathrm{~g}$ & $463 \mathrm{de}$ & $171 \mathrm{~b}$ & $1422 \mathrm{a}$ \\
\hline 6000 & $185 b$ & $23 b$ & $541 d$ & $182 \mathrm{e}$ & $44 \mathrm{e}$ & $85 \mathrm{~d}$ \\
\hline 10000 & $155 b$ & $21 b$ & $765 \mathrm{c}$ & $494 d$ & $258 \mathrm{a}$ & $122 \mathrm{~cd}$ \\
\hline 30000 & $172 b$ & $25 \mathrm{ab}$ & $288 \mathrm{f}$ & $395 \mathrm{e}$ & $27 f$ & $283 b$ \\
\hline 60000 & $182 b$ & $23 b$ & $909 \mathrm{a}$ & $1394 \mathrm{c}$ & $58 \mathrm{de}$ & $257 b$ \\
\hline 90000 & $175 b$ & $22 b$ & $843 b$ & $1594 b$ & $142 \mathrm{bc}$ & $278 b$ \\
\hline 120000 & $262 \mathrm{a}$ & $21 b$ & $297 f$ & $2598 \mathrm{a}$ & $109 \mathrm{~cd}$ & $227 b$ \\
\hline 150000 & $165 b$ & $32 \mathrm{a}$ & $555 \mathrm{~d}$ & $41 \mathrm{f}$ & $41 \mathrm{e}$ & $235 b$ \\
\hline 180000 & $32 \mathrm{c}$ & $31 \mathrm{a}$ & $356 \mathrm{e}$ & $137 \mathrm{e}$ & 74de & $247 b$ \\
\hline \multicolumn{7}{|l|}{ Intemperizado } \\
\hline Testigo & $150 \mathrm{c}$ & $21 \mathrm{a}$ & $199 b$ & $463 b$ & $171 \mathrm{a}$ & $1422 b$ \\
\hline 14173 & $10 \mathrm{e}$ & $14 \mathrm{c}$ & $36 \mathrm{e}$ & $827 \mathrm{a}$ & $31 \mathrm{c}$ & $1633 a$ \\
\hline 28400 & $17 \mathrm{e}$ & $16 \mathrm{~b}$ & $135 \mathrm{~d}$ & $333 \mathrm{c}$ & $79 \mathrm{bc}$ & $1188 b$ \\
\hline 50598 & $77 \mathrm{e}$ & $10 \mathrm{c}$ & $171 \mathrm{c}$ & $216 \mathrm{~d}$ & $125 \mathrm{ab}$ & $629 c$ \\
\hline 75492 & $167 d$ & $11 \mathrm{c}$ & $162 \mathrm{c}$ & $120 \mathrm{e}$ & $99 b$ & $1153 b$ \\
\hline 112142 & $417 \mathrm{a}$ & $12 \mathrm{c}$ & $220 \mathrm{a}$ & $120 \mathrm{e}$ & $116 b$ & $1190 \mathrm{~b}$ \\
\hline
\end{tabular}

*Valores con diferentes letras por columna del mismo color son estadísticamente diferentes (Tukey, $\mathrm{p} \leq 0.05$ ).

*Values with different letters per column of the same color are statistically different (Tukey, $\mathrm{p} \leq 0.05$ ).

(28.4 y 65.2 g) se registró en los tratamientos con las concentraciones de 90000 y 150000 mg. $\mathrm{kg}^{-1}$ b. s. HTPF, es decir un aumento con respecto al suelo testigo de 4.92 y $17.17 \%$ (Fig. 2). Así mismo, los tratamientos con PI, registraron la mayor producción de MS (47.4 y $88.4 \mathrm{~g}$ ) en los tratamientos con 28400 y $112142 \mathrm{mg} \cdot \mathrm{kg}^{-1}$ b. s. HTPI, un aumento del 43.03 y $38.91 \%$ en relación al testigo.

\section{Degradación de petróleo fresco e intem-} perizado: En la figura 3, se observó a los tres y seis meses diferencias estadísticas en los porcentajes de descontaminación de los suelos contaminados con PF y PI con respecto al testigo (Tukey $\mathrm{p} \leq 0.05$ ). A los tres meses, la mayor DSC de suelos se registró en dosis de 150000 mg.kg ${ }^{-1}$ de PF y en 14173 mg.kg-1 b. s. de PI, con 67 y $77 \%$, respectivamente. A los seis meses, la mayor DSC de PF y PI se observó en
6000 y 14173 mg.kg-1 b. s., respectivamente, con $87 \%$ y $86 \%$, pero en PF los suelos con $6000,10000,30000,60000$ y 90000 mg.kg-1 b. s. fueron estadísticamente iguales en el porcentaje de DSC.

\section{DISCUSIÓN}

La investigación reveló un comportamiento variable, tanto en la densidad de BFN como por el tipo de petróleo, aunque es claro que los contenidos de PI estimularon la presencia BFN a concentraciones menores de 50598 mg. $\mathrm{kg}^{-1}$ b. s. HTPI a los seis meses. Esta respuesta probablemente se asocia a que los hidrocarburos del petróleo permiten la disponibilidad de carbono orgánico total y nitrógeno inorgánico en el suelo, los cuales son utilizados como fuente de energía en los procesos metabólicos de las bacterias, lo que permite su 


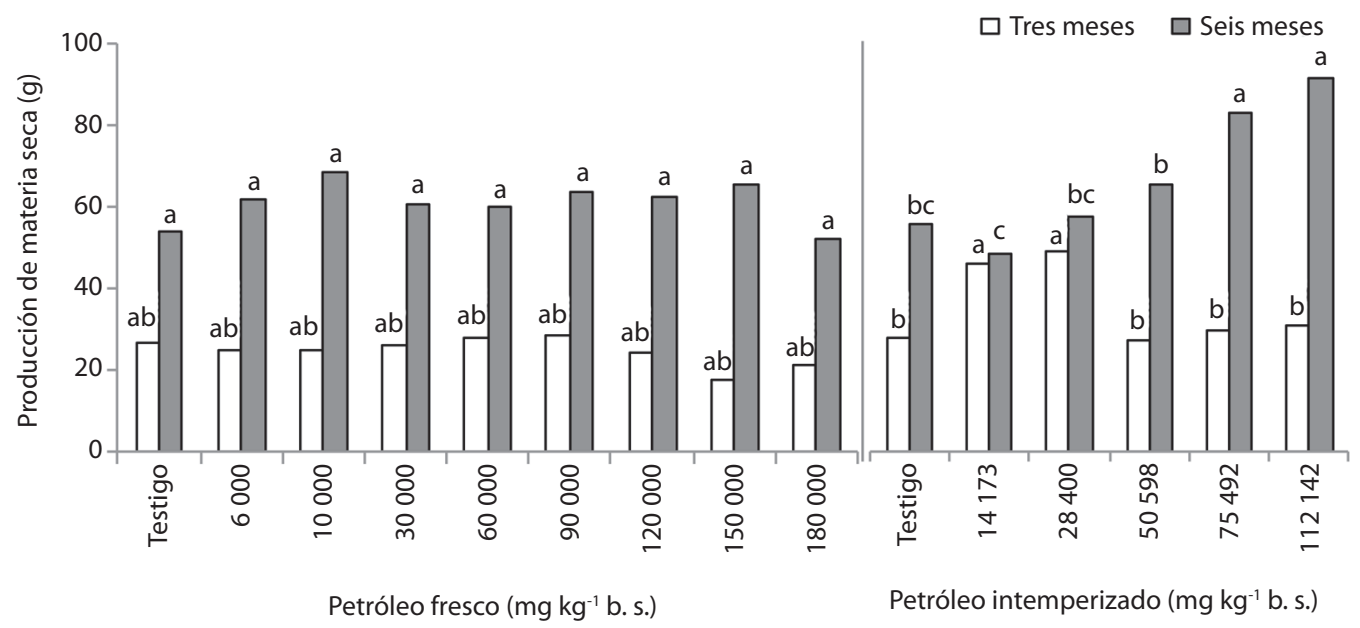

Fig. 2. Producción de materia seca de L. hexandra en suelos contaminados con petróleo fresco e intemperizado, a los tres y seis meses de tratamiento. Valores con diferentes letras por columna del mismo color son estadísticamente diferentes (Tukey, $\mathrm{p} \leq 0.05$ ).

Fig. 2. Dry matter production of $L$. hexandra, in soils contaminated with fresh and weathered oil, for three and six month treatment periods. Values with different letters per column of the same color are statistically different (Tukey, $\mathrm{p} \leq 0.05$ ).

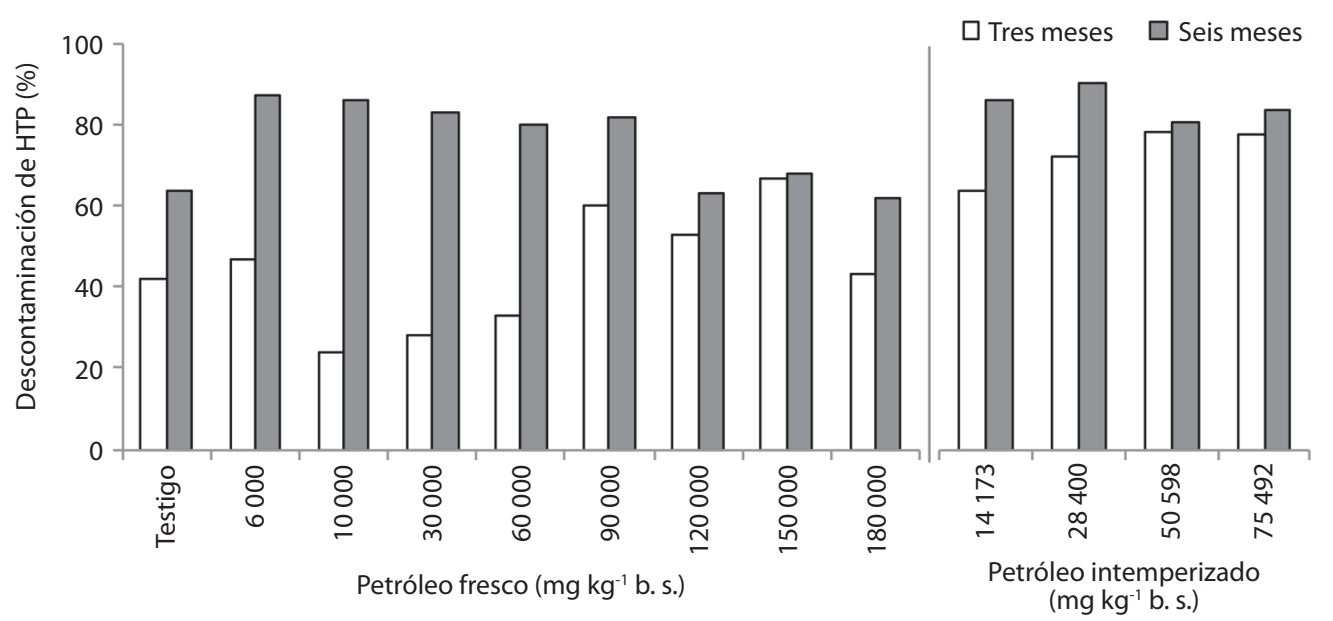

Fig. 3. Descontaminación por L. hexandra (\%) en suelos contaminados con petróleo fresco e intemperizado, a los tres y seis meses de tratamiento.

Fig. 3. L. hexandra decontamination effect (\%) in soils contaminated with fresh and weathered oil for three and six months treatments.

adaptación (Caravaca \& Roldán, 2003; PérezVargas, Anaya-Reza, Chang-Solís, MembrilloVenegas, \& Calva-Calva, 2010). Acuña, Pucci y Pucci (2012) explican que la deficiencia de nitrógeno en suelos produce la disminución de la biomasa microbiana, la mineralización y eliminación de hidrocarburos; esta deficiencia favorece a la degradación de hidrocarburos aromáticos por BFN; por el contrario, la presencia de nitrógeno en el suelo, puede enfocar la degradación de hidrocarburos alifáticos. Sin embargo, sólo un número limitado de especies bacterianas tiene la capacidad combinada de la fijación de nitrógeno y la oxidación de 
hidrocarburos (Trujillo-Narcía et al., 2014). La reducción de las bacterias en la rizósfera de L. hexandra en suelos con PF y PI, puede ser causado por la represión de la síntesis de proteína y DNA de los microorganismos, al estar expuesto a hidrocarburos policíclicos aromáticos como el naftaleno, fluoreno y pireno (Sun, Wang, Sun, Peng, \& Deng, 2012; Penton et al., 2013). Además, el pH es un factor clave para el crecimiento de bacterias nitrificantes, ya que su óptimo crecimiento se encuentra en un $\mathrm{pH}$ neutral o ligeramente alcalino. Por lo tanto, al disminuir el $\mathrm{pH}$ de un suelo a causa de la contaminación por petróleo, se induce a la reducción del número de bacterias nitrificantes (John, Itah, Essien, \& Ikpe, 2011; Zamora, Ramos, \& Arias, 2012).

La producción de materia de $L$. hexandra por encima de los $50 \mathrm{~kg}$, nos indica la capacidad de crecimiento y adaptación a sitios contaminados con hidrocarburos. Una respuesta similar registró Zand, Bidhendi y Mehrdadi (2010) en Zea mays y Festuca arundinacea en suelos con 34358 y 26994 mg.kg-1 b. s. HTP. Vaziri, Panahpour y Mirzaee-Beni (2013) explican que una producción de materia vegetal continua, puede ser efectiva, no solo por la prospección de las raíces, sino por los exudados radicales que mejoran la actividad catabólica de los microorganismos de la rizósfera. Así mismo, las condiciones de anegamiento permiten el aumento de la materia vegetal por el transporte de material fotosintético a las raíces, esto favorece el crecimiento y desarrollo del tejido fotosintético (Liu, Jadeja, Zhou, \& Liu, 2012; Ciotti, Castelán, Hack, Porta, \& González, 2014). El crecimiento y desarrollo vegetal de $L$. hexandra estimuló la actividad metabólica de los microorganimos, que pudo generar el proceso de óxido-reducción de los hidrocarburos del PF y PI en la rizósfera (Khan, Afzal, Iqbal, \& Khan, 2013). El proceso de óxido-reducción se basa en el uso de hidrocarburos como sustrato (donador de electrones) en la respiración de las células microbianas cuyo fin es la producción de biomasa (energía), de modo que la actividad metabólica de la célula acaba degradando y consumiendo dicha sustancia; dependiendo del tipo de aceptores del proceso, este puede denominarse anaerobio $\left(\mathrm{O}_{2}\right)$ o anaerobio $\left(\mathrm{NO}^{3-}, \mathrm{SO}_{4}{ }^{2-}, \mathrm{Fe}^{3+}, \mathrm{Mn}^{4+}\right.$ y $\mathrm{CO}_{2}$ ) (Zambrano \& Araujo, 2015).

Estos resultados indican que la rizosfera de L. hexandra durante los seis meses, estimuló positivamente la producción de materia seca, el establecimiento de bacterias fijadoras de nitrógeno de vida libre, de los grupos Azospirillum y Azotobacter, en dosis altas de petróleo y en condiciones de anegamiento. Además, los porcentajes de descontaminación superiores al $80 \%$, demuestran el potencial fitorremediador de L. hexandra para suelos contaminados con petróleo fresco e intemperizado.

\section{AGRADECIMIENTOS}

Agradecimiento al Colegio de Postgraduados por fideicomiso revocable de administración e inversión No 167304 para el establecimiento y operación de los fondos para la investigación científica y desarrollo tecnológico del centro público. Al CONACYTMéxico por la beca otorgada al M.C. Alfredo Arias Trinidad para realizar su posgrado en el Programa de Producción Agroalimentario en el Trópico del Colegio de Postgraduados, Campus-Tabasco.

\section{RESUMEN}

La industria petrolera ha generado derrames crónicos de petróleo y su acumulación en Gleysoles en zonas anegadas en el estado de Tabasco, en el sureste de México. El anegamiento es un factor que limita el uso de tecnologías de remediación por el alto costo y los bajos niveles de degradación del petróleo, sin embargo, Leersia hexandra Sw. es un pasto que crece en estas zonas contaminadas con petróleo intemperizado. El objetivo del estudio fue evaluar la densidad de bacterias, producción de biomasa vegetal y fitorremediación de L. hexandra en suelo contaminado con petróleos fresco e intemperizado, bajo condiciones experimentales de anegamiento. Se realizaron dos experimentos (E1 y E2) en un túnel de plástico. E1 E1 se basó en ocho dosis: 6000, 10000, 30000, 60000, $90000,120000,150000$ y $180000 \mathrm{mg} \mathrm{kg}^{-1}$ base seca (b. s.) de hidrocarburos totales de petróleo fresco (HTPF), y en el E2 se evaluaron cinco dosis: 14173, 28400, 50598, 75492 y $112142 \mathrm{mg} \mathrm{kg}^{-1}$ b. s. de hidrocarburos totales de petróleo intemperizado (HTPI), con ocho repeticiones en 
cada experimento, además se utilizó un testigo con 2607 $\mathrm{mg} \mathrm{kg}^{-1}$ b. s. de HTP de origen biogénico. Las variables evaluadas a los tres y seis meses fueron 1) densidad microbiana de las bacterias fijadoras de nitrógeno de vida libre totales (BFN), del grupo Azospirillum (AZP) y Azotobacter (AZT), por cuenta viable en placa seriada; 2) producción de materia seca total (MS), se cuantificó por el peso seco por gravimetría, y 3) el porcentaje de descontaminación de los hidrocarburos (DSC) por extracción en equipo soxhlet. En suelos con HTPF, la población de BFN, AZP y AZT se estimuló hasta cinco veces más que el tratamiento testigo a los tres y seis meses; sin embargo, concentraciones de 150000 y $180000 \mathrm{mg} \mathrm{kg}^{-1}$ b. s. inhibieron entre un 70 y $89 \%$ la densidad bacteriana. A su vez, en suelos con PI, la inhibición se registró hasta en un $90 \%$, a excepción del tratamiento con $14173 \mathrm{mg} \mathrm{kg}^{-1}$ b. s., el cual estimuló las BNF y AZT en 2 y 0.10 veces más que testigo, respectivamente. La producción de MS fue continua en los experimentos hasta los seis meses, con valores de 63 y 89 g en PF y PI, respectivamente; sin diferencias significativas con el testigo $(\mathrm{p} \leq 0.05)$. El DSC alcanzó valores del $66 \%$ al $87 \%$ en HTPF como HTPI a los seis meses, respectivamente. Estos resultados demuestran la habilidad del L. hexandra para desarrollar una rizósfera con alta densidad de BFN, producir biomasa vegetal y fitorremediar Gleysoles con petróleo fresco e intemperizado en ambientes tropicales inundados.

Palabras clave: Azotobacter, Azospirillum, pasto tropical, petróleo intemperizado, fitorremediación.

\section{REFERENCIAS}

Acuña, A. J., Pucci, O. H., \& Pucci, G. N. (2012). Effect of nitrogen deficiency in the biodegradation of aliphatic and aromatic hydrocarbons in patagonian contaminated soil. International Journal of Research and Reviews in Applied Sciences, 11(3), 470-476.

Aparicio, R., González-Ronquillo, M., Torres, R., Astudillo, L., Córdova, L., \& Carrasquel, J. (2007). Degradabilidad de los pastos lambedora (Leersia hexandra) y paja de agua (Hymenachne amplesicaulis) en cuatro épocas del año de una sábana inundable del estado Apure, Venezuela. Zootecnia Tropical, 25(3), 225-228.

Bisht, S., Pandey, P., Bhargava, B., Sharma, S., Kumar, V., \& Sharma, K. D. (2015). Bioremediation of polyaromatic hydrocarbons (PAHs) using rhizosphere technology. Brazilian Journal of Microbiology, 46(1), 7-21.

Capó, M. A. (2007). Principios de ecotoxicología: diagnóstico, tratamiento y gestión del medio ambiente. Madrid, España: Editorial Tebar.

Caravaca, F., \& Roldan, A. (2003). Assessing changes in physical and biological properties in a soil contaminated by oil sludges under semiarid Mediterranean conditions. Geoderma, 117(1), 53-61.

Chávez-Rodríguez, L. (2015). Phytoremediation of lead polluted soils with native plant species. IOSR Journal of Environmental Science, Toxicology and Food Technology, 9(4), 42-49.

Ciotti, E. M., Castelán, M. E., Hack, C. M., Porta, M., \& González, A. M. (2014). Tolerancia de leguminosas herbáceas estivales a condiciones de anegamiento temporal. Tropical Grasslands, 2(1), 278-286.

Döbereiner, J., Marriel, I. E., \& Nery, M. (1966). Ecological distribution of Spirillum lipoferum Beijerinck. Canadian Journal Microbiology, 22(10), 1464-1473.

DOF (2002). Norma Oficial Mexicana NOM-021-RECNAT-2000, que establece las especificaciones de fertilidad, salinidad y clasificación de suelos. Estudios, muestreo y análisis. Diario Oficial de la Federación. D.F., México. http://www.profepa.gob.mx/ innovaportal/file/3335/1/nom-021-semarnat-2000. pdf. Fecha de consulta 14 de agosto del 2014.

DOF (2006). Norma Mexicana NMX-AA-134-SCFI2006, Suelos. Hidrocarburos Fracción Pesada por Extracción y Gravimetría. Método De Prueba. Diario Oficial de la Federación. D.F., México. http://legismex.mty.itesm.mx/normas/aa/nmx-aa-06/proy-nmxaa-134-scfi-2006.pdf. Fecha de consulta 14 de agosto del 2014.

DOF (2013). Norma Oficial Mexicana NOM-138-SEMARNAT/SAI-2012, Límites máximos permisibles de hidrocarburos en suelos y lineamientos para el muestreo y la remediación. Diario Oficial de la Federación. D.F., México. http://www.dof.gob.mx/nota detalle.php? codigo $=5313544 \&$ fecha $=10 / 09 / 2013$. Fecha de consulta 14 de agosto del 2014.

Fernández, C., Silva, M., Pereira, J. C., Mallia, A., Llobregat, M. J., \& Atomare, V. (2006). Biodegradabilidad de las fracciones de resinas y asfáltenos por pseudomonas en suelo impactado con petróleo crudo mediano. Ingeniería Universidad de Carabobo, 13(4), 7-13.

García-López, E., Zavala-Cruz, J., \& Palma-López, D. J. (2006). Caracterización de las comunidades vegetales en un área afectada por derrames de hidrocarburos. Terra Latinoamericana, 24(1), 17-26.

Hernández-Castellanos, B., Zavala-Cruz, J., Martínez-Hernández, S., Dendooven, L., Contreras-Ramos, S. M., Noa-Carrazana, J. C., Fragoso, C., \& Ortíz-Ceballos, A. I. (2013). Earthworm Population in an Aged Hydrocarbon Contaminated Soil. Research Journal of Environmental Sciences, 7(1), 27-37.

Hernández-Rivera, M. A., Ojeda-Morales, M. E., Martínez-Vázquez, J. G., Villegas-Cornelio, V. M., \& Córdova-Bautista, Y. (2011). Optimal parameters fort In Vitro development of the hydrocarbonoclastic 
microorganism Proteus sp. Journal of Soil Science and Plant Nutrition, 11(1), 29-43.

John, R. C., Itah, A. Y., Essien, J. P., \& Ikpe, D. I. (2011). Fate of nitrogen-fixing bacteria in crude oil contaminated wetland Ultisol. Bulletin of Environmental Contamination and Toxicology, 87(3), 343-353.

Khan, S., Afzal, M., Iqbal, S., \& Khan, Q. M. (2013), Plant-bacteria partnerships for the remediation of hydrocarbon contaminated soils. Chemosphere, 90(4), 1317-1332.

Liu, J., Duan, C., Zhang, X., Zhu, Y., \& Xiaoyan, L. (2011). Potential of Leersia hexandra Swartz for phytoextraction of Cr from soil. Journal of Hazardous Materials, 188(20), 85-91.

Liu, R., Jadeja, R. N., Zhou, Q., \& Liu, Z. (2012). Treatment and remediation of petroleum-contaminated soils using selective ornamental plants. Environmental Engineering Science, 29(6), 494-501.

Lum, A. F., Ngwa, E. S., Chikoye, D., \& Suh, C. E. (2014). Phytoremediation potential of weeds in heavy metal contaminated soils of bassa industrial zone of Doulala. International Journal of Phytoremediation, 16(3), 302-319.

Madigan, M. T., Martinko, J. M., Dunlap, P. V., \& Clark, P. D. (2009). Brock, Biología de los Microorganismos. $12^{\mathrm{a}}$ ed. España: Pearson Educación.

Maletic, S. P., Dalmacija, B. D., Roncevic, S. D., Agbaba, J. R., \& Garcina, S. D. U. (2011). Impact of hydrocarbon type, concentration and weathering on its biodegradability in soil. Journal of Environmental Science and Health, 46(10), 1042-1049.

Mganga, N. D. (2014). The potential of bioaccumulation and translocation of heavy metals in plant species growing around the tailing dam in Tanzania. International Journal of Science and Technology, 3(10), 690-697.

OECD (2003). Guideline for the testing of chemicals proposal for updating guideline 208; terrestrial plant test: seedling emergence and seedling growth test. Organization for Economic Co-operation and Development. http://www.oecd-library.org/docserver/ download/9720801e.pdf?expires $=1427427662 \&$ id $=$ id\&accname $=$ guest \& checksum $=7$ A2 7257197 E7341 7A573AD35039252B4. Fecha de consulta 03 de febrero del 2015.

OMI (2005). Manual sobre la contaminación ocasionada por los hidrocarburos: parte IV, lucha contra los derrames de hidrocarburos. 2a ed. Reino Unido: Organización Marítima Internacional.

Paz-Alberto, A. M., \& Sigua, G. C. (2013). Phytoremediation: a Green technology to remove environmental pollutants. American Journal of Climate Change, 2(1), 71-86.
PEMEX (2016). Indicadores petroleros 2014-2016. Petroleos Mexicanos. http://www.pemex.com/ri/Publicaciones/Paginas/IndicadoresPetroleros.aspx

Penton, C. R., Johnson, T. A., Quensen, J. F., Iwai, S., Cole, J. R., \& Tiedje, J. M. (2013). Functional genes to assess nitrogen cycling and aromatic hydrocarbon degradation: primers and processing matter. Frontiers in Microbiology, doi:10.3389/fmicb.2013.00279.

Peña, W., Trasar-Cepeda, C., Gil-Sotres, F., \& Leirós, M. C. (2007). Modification of the degradative capacity of a soil artificially contaminated with diesel. Chemosphere, 67(5), 1057-1063.

Pérez-Vargas, J., Anaya-Reza, O., Chang-Solís, C. K., Membrillo-Venegas, I. L., \& Calva-Calva, G. (2010). Producción de biosurfactantes por bacterias de vida libre fijadoras de nitrógeno crecidas en hidrocarburos. Revista Centro Nacional de Investigaciones Cientificas Ciencias Químicas, 41(1), 1-9.

PROFEPA (2014). Las emergencias ambientales en México: consecuencias e impactos. http://www.cenapred. gob.mx:8080/SeminarioInternacional2014/documentos/mesa3 emergencias.pdf

Rainho, C. R., Corrêa, S. M., Mazzei, J. L., Aiub, C. A. F., \& Felzenszwalb, I. (2013). Genotoxicity of polycyclic aromatic hydrocarbons and nitro-derived in respirable airborne particulate matter collected from urban áreas of Rios de Janeiro (Brazil). Biomed Research International, doi:10.1155/2013/765352

Reinhold, B., Hurek, T., Fendrik, I., Pot, B., Gillis, M., Kersters, K., Thielemans, D., \& De Ley, J. (1987). Azospirillum halopraeferans sp. nov., a nitrogen fixing organism associated with roots of Kallar (Leptochloa fusca (L) Kunth). International Journal Systematic Bacteriology, 37(1), 43-46.

Rennie, R. J. (1981). A single medium for the isolation of acetylene-reducing (dinitrogen-fixing) bacteria from soils. Canadian Journal Microbiology, 27(1), 8-14.

Rivera-Cruz, M. C., \& Trujillo-Narcia, A. (2004). Estudio de toxicidad vegetal en suelos contaminados con petróleos nuevo e intemperizado. Interciencia, 29(7), 369-376.

Shao-Hong, Y., Xue-Hong, Z., Jie, L., Yi-Nian, Z., \& Chen, G. (2013). Feasibility of constructed wetland planted with Leersia hexandra Swartz for removing $\mathrm{Cr}, \mathrm{Cu}$ and $\mathrm{Ni}$ from electroplating wastewater. Environmental Technology, 35(2), 187-194.

Sun, F. L., Wang, Y. S., Sun, C. C., Peng, Y. L., \& Deng, C. (2012). Effects of three different PAHs on nitrogenfixing bacterial diversity in mangrove sediment. Ecotoxicology, 21(6), 1651-1660.

Trujillo-Narcía, A., Rivera-Cruz, M. C., Lagunes-Espinoza, L. C., Palma-López, D. J., Sánchez-Soto, S., \& Ramírez-Valverde, G. (2014) Biological parameters 
of the restoration of soil polluted by crude oil. Ecosistemas y Recursos Agropecuarios, 1(2), 107-122.

Uren, C. N. (2001). Types, Amounts, and Possible Functions of Compounds Released into the Rhizosphere by Soil-Grown Plants. In R. Pinton, Z. Varanini, \& P. Nannipieri (Eds.), The Rhizosphere: Biochemistry and Organic Substances at the SoilPlant Interface (pp. 42-120). New York, American United States: CRC Press.

Vaziri, A., Panahpour, E., \& Mirzaee-Beni, M. H. (2013). Phytoremediation, a method for treatment of petroleum hydrocarbon contaminated soils. International Journal of Farming and Allied Sciences, 2(21), 909-913.

Wang, J., Zhan, X., Zhou, L., \& Lin, Y. (2010). Biological Indicators capable of assessing thermal treatment efficiency of hydrocarbon mixture contaminated soil. Chemosphere, 80(8), 837-844.
Zambrano, K., \& Araujo, I. (2015) Tratamiento Biológico de Sedimentos Contaminados con Hidrocarburos. Madrid: Editorial Académica Española.

Zamora, A., Ramos, J., \& Arias, M. (2012). Efecto de la contaminación por hidrocarburos sobre algunas propiedades químicas y microbiológicas de un suelo de sabana. Bioagro, 24(1), 5-12.

Zand, A. D., Bidhendi, G. N., \& Mehrdadi, N. (2010). Phytoremediation of total petroleum hydrocarbons (TPHs) using plant species in Iran. Turkish Journal of Agriculture and Forestry, 34(5), 429-438.

Zavala-Cruz, J., Trujillo-Capistrán, F., Ortiz-Ceballos, G. C., \& Ortiz-Ceballos, A. I. (2013) Tropical endogeic earthworm population in a pollution gradient with weathered crude oil. Research Journal of Enviromental Sciences, 7(1), 15-26. 